Escuela de Ciencias Sociales, Artes y Humanidades

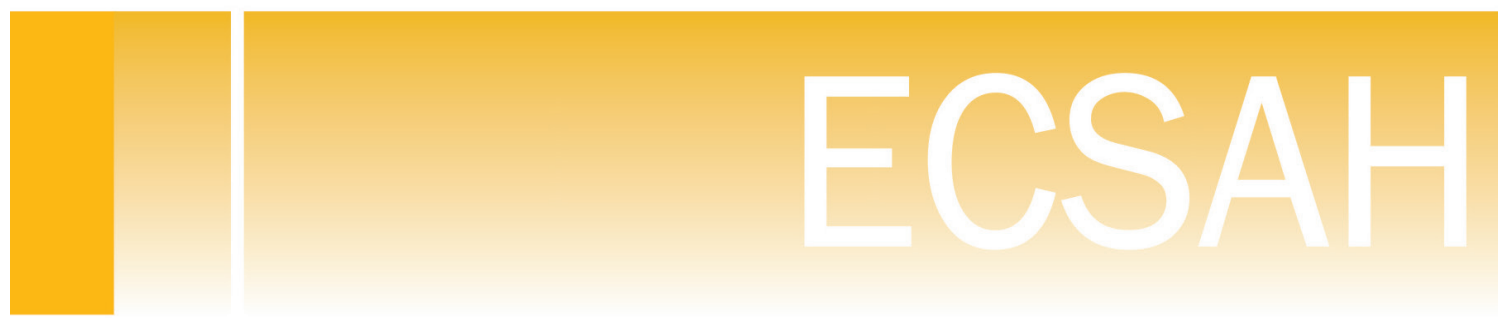





\title{
DIAGNÓSTICO PSICOSOCIAL DE LA JUVENTUD DE ZIPAQUIRÁ: UN ANÁLISIS DESDE LA COMUNICACIÓN
}

\author{
Sonia Bibiana Rojas, ${ }^{46}$ Diana F. Scarpeta Rondón, ${ }^{47}$ \\ Doris Margarita Algarra Echenique, ${ }^{48}$ Cecilia Durán Avendaño, ${ }^{49}$ \\ Sandra Patricia Valbuena ${ }^{50}$ y Sandra Patricia Jiménez ${ }^{51}$
}

\begin{abstract}
RESUMEN
La ponencia presenta los resultados preliminares del proyecto Diagnóstico Psicosocial de la Juventud de Zipaquirá: Una base para la Formulación de la Política Pública Municipal de Juventud, específicamente en la Línea de Comunicación, la cual se ha convertido en un eje transversal de la investigación por cuanto la comunicación se empieza a evidenciar como dispositivo fundamental para el desarrollo psicosocial de los jóvenes.

Se trabajó con un enfoque participativo de investigación utilizando técnicas mixtas. En general, los resultados preliminares reflejan la percepción propia del grupo de los jóvenes y otros actores sociales frente a los problemas, necesidades y potencialidades de la juventud de Zipaquirá, así como las fortalezas de la oferta institucional municipal en cuanto al tema de comunicación, acceso a la información, visibilidad y empoderamiento juvenil.
\end{abstract}

Palabras clave: comunicación, información, visibilidad, empoderamiento, juventud, Política Pública.

\begin{abstract}
This paper presents the preliminary results of the project "Psychosocial Diagnosis for The Zipaquirá Youth: A base to formulate a Municipal Public Policy for Youth", specifically on the Communication Line, which has become a cross transverse axis of this Investigation, since the communication is started demonstrating as fundamental device for the development psychosocial of the young people. It was worked with a participatory approach of investigation using mixed techniques. In general, preliminary results reflect the group's own perception of young men and women and other social actors address the problems, needs and

\footnotetext{
46 Coinvestigador Grupo de investigación Chicaquicha UNAD.

47 Líder del Grupo de Investigación Chicaquicha. unAD. Código de registro del Grupo en Colciencias: COL0091045, Línea de Investigación: contextos socioculturales, procesos psicosociales, comunitarios y Psicología Social Comunitaria. Red de Investigación: Desarrollo Comunitario, Participación y Equidad. UNAD.

48 Coinvestigador Grupo de investigación Chicaquicha UNAD.

49 Coinvestigador Grupo de investigación Chicaquicha UNAD.

50 Auxiliar de investigación Chicaquicha, UNAD.

51 Auxiliar Grupo de investigación Chicaquicha UNAD
} 
potentials of youth from Zipaquirá, as well as the strengths of local institutional offer on the communication subject, access to information visibility and youth empowerment.

Key words: communication, information, visibility, empowerment, youth, Public Policy.

\section{INTRODUCCIÓN}

Los resultados que se presentan en esta ponencia hacen parte de los avances obtenidos hasta el momento en la investigación Diagnóstico Psicosocial de la Juventud de Zipaquirá: Una base para la Formulación de la Política Pública Municipal de Juventud, desarrollada por el Grupo de Investigación Chicaquicha como proyecto aprobado en la convocatoria interna de SIUNAD 2009. Su objetivo es el de identificar el estado actual de la realidad psicosocial de los jóvenes entre 14 y 26 años del Municipio de Zipaquirá, mediante el desarrollo de metodologías de diagnóstico participativas, enfocadas en las líneas estratégicas orientadoras para la formulación de la Política Municipal de Juventud. Estas líneas son retomadas de la Política Pública de Juventud de Cundinamarca promulgada a través de la Ordenanza 020 de 2007, como ejes de análisis que han permitido conocer los problemas, necesidades y potencialidades de la juventud de Zipaquirá, así como las fortalezas de la oferta institucional municipal, en temas como: convivencia y familia, educación, salud, cultura e identidad, emprendimiento y condiciones laborales, organización y participación juvenil, medio ambiente, y comunicación.

Este último tema se ha venido abordando como una línea independiente a partir de tres categorías de análisis: medios de comunicación, información y visibilidad. No obstante, en el transcurso de la investigación se ha convertido en un eje transversal por cuanto la comunicación se empieza a evidenciar como dispositivo fundamental para el desarrollo psicosocial de los jóvenes: la información y la tecnología se constituyen en elementos que les permite visibilizarse, propiciar su reconocimiento, el fortalecimiento de sus identidades y su inclusión social. Es en este punto que las formas de comunicación se convierten en elementos centrales en los cambios juveniles, en tanto en palabras de Barbero (1998: p. 24) "viven una experiencia de desanclaje" que despiertan sensibilidades en los jóvenes apartándolos de los estilos y prácticas tradicionales y acercándolos a conexiones relacionadas con la informática, la imagen, el sonido, promoviendo un espacio comunicacional propio y colectivo a partir de formas de ver, sentir, oír e interpretar la realidad.

Los argumentos, justifican el interés de centrar esta ponencia, en el análisis de los resultados de esta línea específica, que se ha convertido en el proyecto de grado de dos estudiantes de Psicología de la UNAD, auxiliares de investigación del Diagnóstico Psicosocial. En principio, es importante resaltar que tanto el proyecto global como el abordaje de la Línea de Comunicación se enmarcan teóricamente en el enfoque del Empoderamiento que surge de la Psicología Social Comunitaria e implica un "proceso mediante el cual las personas, las organizaciones y las comunidades ganan control sobre sus vidas" (Rappaport, 1984; citado por Silva y Loreto). Desde este enfoque se tienen como prioridades la movilización, la educación, la autogestión, y 
toda serie de prácticas sociales encaminadas a devolver el protagonismo a los jóvenes en la construcción de su propio desarrollo.

En ese sentido, los medios de comunicación se convierten en instrumentos fundamentales para el empoderamiento juvenil, pues no sólo el acceso a la información que estos proporcionan, sino su apropiación crítica, permite a la juventud convertirse en sujeto activo en la construcción de su propia identidad, utilizando estos medios como herramientas para aumentar la capacidad de comprensión y transformación de su realidad concreta.

\section{OBJETIVO GENERAL}

Identificar con los jóvenes las principales necesidades y problemas que les afecta en relación con el acceso a la información y apropiación de los medios de comunicación.

\section{OBJETIVO ESPECÍFICOS}

Determinar cuáles son los medios de comunicación más utilizados por la juventud del municipio y la manera como los jóvenes se visibilizan a través del acceso y apropiación de estos medios.

Diseñar una propuesta orientada al empoderamiento juvenil en los medios de comunicación e información que les permita generar espacios de participación y fortalecimiento de la identidad que los medios puedan aportar.

\section{METODOLOGÍA}

El proyecto se ha venido desarrollando desde un enfoque participativo de investigación, el cual se caracteriza porque "posee un interés por comprender la realidad social y material de aquellos que la viven cotidianamente, pero no es su pretensión última, (...) puesto que no se queda con la comprensión sino que toma ésta como medio para orientar la planeación de la acción social" (López, 2001: 143). Por tanto, este tipo de investigación permite un acercamiento a la realidad juvenil desde la perspectiva misma de las y los jóvenes, articulando el saber académico y el saber social, con miras a la transformación de sus problemas y el desarrollo psicosocial de esta población.

Se utiliza un diseño metodológico mixto que se hace operativo a partir de la aplicación de técnicas cualitativas y cuantitativas así:

\section{- Encuesta para el diagnóstico de la juventud del municipio de Zipaquirá - 2009:}

Participantes. Universo: población total del Municipio (142.835 Habitantes) Población: Jóvenes entre 14 y 26 años (31.274) De acuerdo con el sexo: mujeres: 15.634, Hombres: 15.640. Muestra: 3.533 jóvenes entre 14 y 26 años del sector rural y urbano del Municipio de Zipaquirá. Se trabajó con un nivel de confianza del 98.5\%. 
Tipo de encuesta: encuesta estructurada compuesta por 65 ítems de tipo cerrado, 5 de ellos correspondientes a la Línea de Comunicación.

Trabajo de Campo: la recolección de la información se llevó a cabo durante los meses de Septiembre, Octubre y Noviembre del 2009. La aplicación fue realizada en instituciones de educación media y superior, instituciones de educación técnica y tecnológica, instituciones de educación no formal, puerta a puerta en sectores del área rural y urbana; sujetos seleccionados aleatoriamente en parques, plazas y centros de congregación juvenil.

Análisis estadístico: se aplicó una estadística descriptiva e inferencial.

\section{- Cartografía Social}

Participantes: 34 jóvenes entre 14 y 26 años residentes en el municipio y que se destacaron por su liderazgo e interés durante la etapa de aplicación de la encuesta.

Metodología: realización de un taller de cartografía social en las instalaciones de la Biblioteca Pública Regional del Municipio de Zipaquirá con el siguiente protocolo: 1. Sensibilización, 2. Explicación de la técnica, 3. Producción cartográfica, 4. Socialización de los resultados, 5. Conclusiones y cierre.

\section{- Entrevista a actores clave}

Participantes: se realizó un total de tres entrevistas.

Metodología: esta técnica fue implementada a través de la aplicación de cuestionarios semiestructurados con preguntas de tipo abierto, a actores clave que fueron seleccionados de acuerdo con su trayectoria y experiencia en el tema de juventud y comunicación en el municipio de Zipaquirá.

Resultados: tanto para la cartografía social como para las entrevistas, se utiliza la técnica de análisis de contenido, agrupando la información en matrices descriptivas. Los resultados se presentan de acuerdo con cada categoría de estudio teniendo en cuenta las variables: Problemas, necesidades psicosociales, fortalezas y soluciones.

\section{RESULTADOS PARCIALES}

El desarrollo del proyecto de investigación se ha planteado en cuatro fases: 1. Fase de planificación de la investigación, 2. Fase Diagnóstica, 3. Fase de Sistematización de la Información y 4. Fase de conclusiones y propuestas. En ese momento el proyecto se encuentra en su tercera fase: luego de la recopilación de datos, se apunta a su ordenamiento, a encontrar relaciones entre ellos y a descubrir la coherencia interna de los diferentes procesos desarrollados en el diagnóstico. A continuación se presentan los resultados parciales de este proceso para la Línea de Comunicación. 


\section{- Resultados Encuesta}

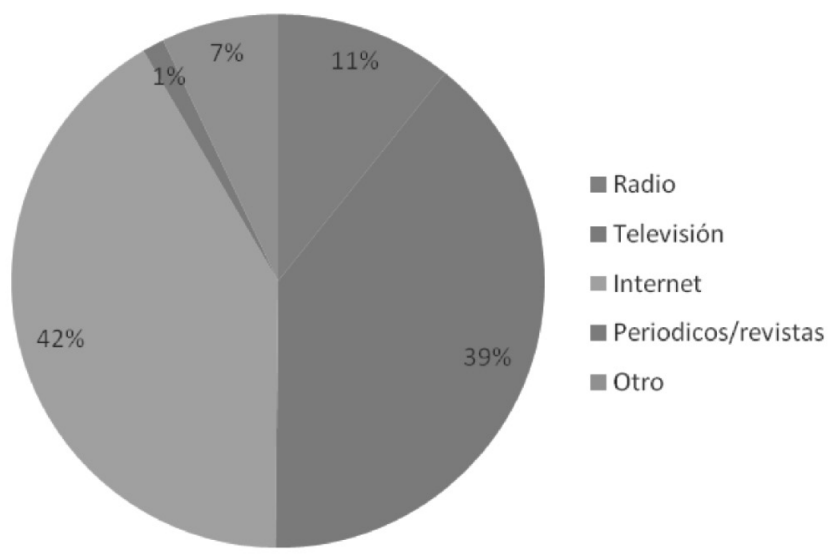

Gráfica 1. Utilización de medios de comunicación.

42. ¿Cuál es el medio de comunicación que más utilizas? (Pregunta cerrada - Única respuesta)

Aunque se evidencia que aún los jóvenes hacen un uso frecuente de la televisión, le sigue de cerca la Internet con un $42 \%$, lo cual demuestra la preferencia por las nuevas tecnologías para acceder a la información, como forma de comunicarse y de ocupar su tiempo libre. Se observa la poca utilización de medios tradicionales como el periódico y la radio.

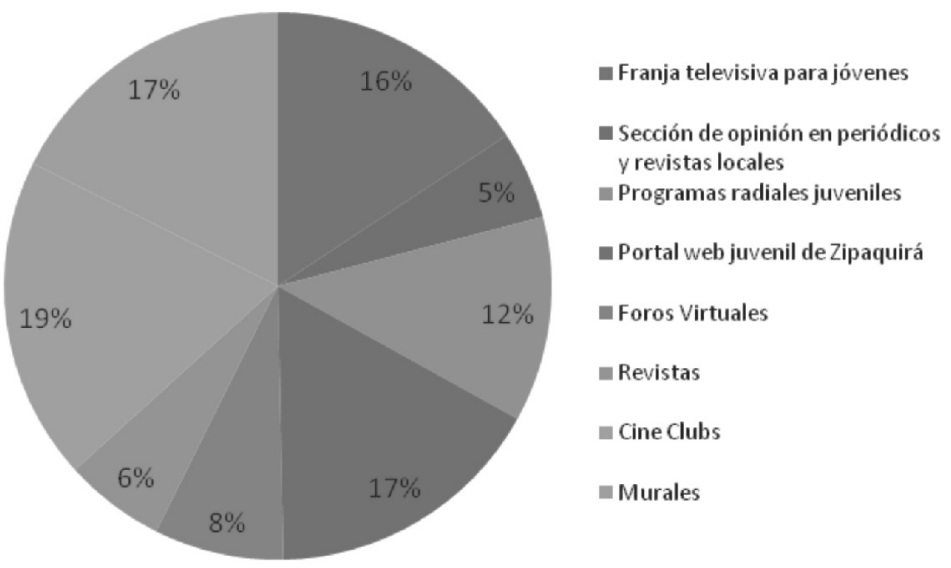

Gráfica 2. Medios y espacios de comunicación que les gustarían a los jóvenes 43. ¿Qué medios y espacios de comunicación para la juventud te gustaría que fueran implementados en Zipaquirá? (Pregunta cerrada - Única respuesta)

Se hace evidente el interés que los jóvenes manifiestan en la implementación de nuevos medios de comunicación. Al analizar los resultados se observa que el 19\% de los jóvenes 
desearía que se implementaran cineclubs, seguido por los murales y portal web juvenil con un $17 \%$ y franja televisiva para jóvenes con un $16 \%$.

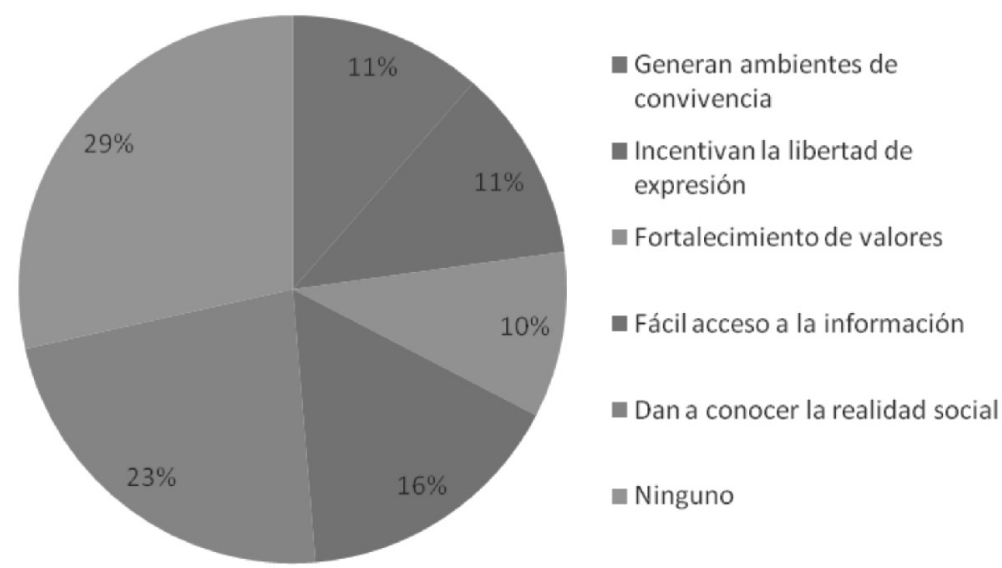

Gráfica 3. Aporte de los medios de comunicación a la juventud

44. ¿Cuáles son los aportes más importantes de los medios de comunicación al Municipio de Zipaquirá? (Pregunta cerrada - Única respuesta)

Un importante porcentaje de los jóvenes entrevistados (29\%) considera que los medios de comunicación no generan aportes significativos al municipio, frente a un $23 \%$ que considera aportan en el conocimiento de la realidad social. Por otro lado, casi en iguales proporciones (10\% y $11 \%)$ se encuentra: el fortalecimiento de valores, incentivan la libertad de expresión y generan ambientes de convivencia.
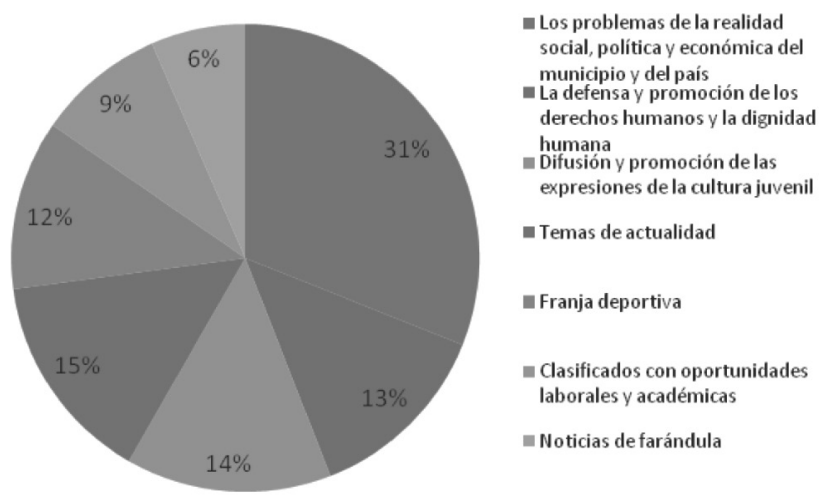

Gráfica 4. Temáticas de interés en los medios de comunicación

45. Si hicieras parte de algún medio de comunicación: ¿Qué temáticas te gustaría trabajar?

(Pregunta cerrada - Múltiple respuesta)

Se puede evidenciar en los jóvenes un mayor interés en los problemas sociales, políticos y económicos del municipio y país, el 31\% refieren esta como temática a trabajar en los 
diferentes medios de comunicación, ya en porcentajes más bajos se encuentran temas de actualidad (15\%), difusión y promoción de expresiones de la cultura (13\%), y la defensa y promoción de los derechos humanos.

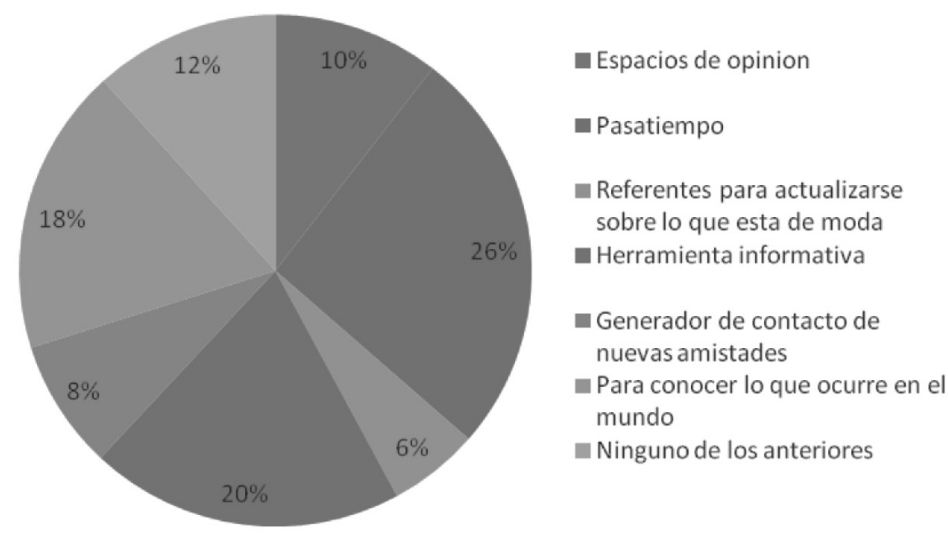

Gráfica 5. Utilización de los medios de comunicación

46. Utilizas los medios de comunicación como: (Pregunta cerrada - Única respuesta)

Se observa que la opinión en los jóvenes se encuentra bastante dividida acerca de la utilización de los medios de comunicación, pues el $26 \%$ lo usa como un pasatiempo, mientras un $20 \%$ la considera aún como una herramienta que les brinda información siendo congruente con el $18 \%$ que buscan conocer por medio de estos lo que ocurre en el mundo. Aún persiste el bajo nivel (10\%) para percibir a los medios de comunicación como espacios para expresar sus opiniones.

\section{RESULTADOS TÉCNICAS CUALITATIVAS}

\section{Problemáticas}

A través de la información recopilada se evidencia que la baja participación de los jóvenes en el manejo de los medios de comunicación se debe, por un lado, a que nos se les proporciona la suficiente información, y por otro, a que no se les incentiva a trabajar en estos espacios.

De otro lado, los jóvenes participantes del proyecto consideran que existe una importante oferta en el municipio en términos de programas y proyectos para la juventud. No obstante, consideran que la divulgación de los mismos es muy escasa, no existen medios apropiados que permitan una amplia difusión de información sobre esta oferta, la publicidad es poco atractiva para los jóvenes, cuestión esta, que sumada a la apatía y poca participación juvenil, hace que muchos de estos procesos fracasen.

\section{Necesidades psicosociales}


Una de las principales necesidades identificadas por las y los jóvenes fue la de implementar espacios de comunicación e información en ámbitos de interacción formativa: escuelas, universidades, academias, agrupaciones, comunidades, familia. Fue una afirmación constante que los jóvenes necesitan espacios en los cuales se pueda desarrollar e incentivar la creatividad, la reflexión, la invención y el diseño de propuestas para el desarrollo juvenil, que se puedan divulgar y visibilizar a través de los medios de comunicación locales.

\section{Fortalezas}

Se evidencia que en el municipio existen medios de comunicación locales como el canal APRECUZ, que permite a los jóvenes informarse y conocer las problemáticas de su entorno. Asimismo, espacios como las emisoras de instituciones educativas, periódicos locales y redes sociales virtuales, se convierten en medios de comunicación, acceso a la información y visibilidad para los jóvenes, donde éstos desempeñan un papel fundamental en el fortalecimiento de su identidad. Un ejemplo concreto es el periódico Ágora de Zipaquirá que ofrece una franja importante para los jóvenes y sus expresiones culturales.

\section{Soluciones}

Los jóvenes consideran que para integrar los medios de comunicación a su desarrollo psicosocial se deben hacer propuestas interesantes para ellos, donde su voz sea realmente tenida en cuenta, espacios donde puedan decir lo que están pensando, lo que ellos quieren ser o no ser.

Frente al problema de difusión de los eventos, espacios, programas y proyectos para la juventud, al igual que para la divulgación de actividades recreo- deportivas y culturales, las y los jóvenes proponen que se implemente una publicidad más amplia, impactante y atractiva, que haga uso de estrategias como el teatro, el video y la música; la creación de páginas web y emisoras juveniles como espacios para la promoción de sus iniciativas y el aprovechamiento de redes sociales virtuales para difundir información sobre la oferta cultural del municipio.

\section{PERSPECTIVAS DEL TRABAJO}

A partir de los resultados del diagnóstico, se busca establecer una línea de base para la formulación de la Política Pública Municipal de Juventud. Esto se logrará a través de la aplicación de dos técnicas: el Metaplán y la Matriz de Marco Lógico.

El Metaplán es una herramienta con enfoque metodológico social participativo, que facilitará realizar una moderación grupal, para dinamizar las diferentes percepciones que posen los jóvenes, frente a las líneas estratégicas de desarrollo juvenil. Posibilita la creación de un escenario de construcción conjunta, para detectar componentes y soluciones factibles a los problemas planteados. 
En el desarrollo del Metaplán se identificarán los problemas principales dentro de cada línea estratégica, que serán presentados a los jóvenes líderes identificados durante el proceso, quienes participarán reconociendo las casusas de cada problema y los efectos del mismo, permitiendo así la integración con el desarrollo de una matriz de marco lógico, con la cual se identificarán los propósitos, los fines, objetivos y proyectos que se podrían desarrollar en cada línea, definiendo alternativas de solución como base para la formulación de la Política Pública Municipal de Juventud y su derivación en un Plan de Desarrollo Municipal de Juventud.

Específicamente en la Línea de Comunicación y con base en los resultados del proyecto el Grupo de Investigación Chicaquicha espera diseñar e implementar una propuesta orientada al empoderamiento juvenil en los medios de comunicación e información que les permita generar espacios de participación y fortalecimiento de la identidad que los medios puedan aportar.

\section{DISCUSIÓN}

Los resultados de la investigación muestran que el uso de medios de comunicación como la Internet, se hacen cada vez más frecuentes entre los jóvenes. A diferencia de la televisión, que implica un consumo transgeneracional, el acceso a la cultura virtual representa un consumo marcado por la brecha generacional.

El vínculo de los jóvenes con las Nuevas Tecnologías de la Comunicación marca una diferencia cognitiva y perceptiva con respecto al mundo de los adultos, dado que en promedio la conectividad de los jóvenes es mucho mayor que la de la población de 30 años y más de edad. Desafortunadamente, en gran medida el acceso a estos espacios y tecnologías de la información se hace de manera pasiva, donde la juventud se convierte tan solo en receptora de información, papel que le otorgan los medios.

En ese sentido, multiplicidad de sectores juveniles permanecen al margen de los beneficios y ventajas asociados a las TIC, no sólo por la falta de acceso a estas tecnologías sino por la dificultad, aún latente, que poseen para transformar el cúmulo de información que circula en estos medios, en conocimiento efectivo para la comprensión y transformación de y desde su realidad. Se trata de un escenario de transferencia y aprendizaje de conocimientos tecnológicos, que solo será posible mediante la incitación de la curiosidad, la investigación y la creatividad a través de la educación.

Por tanto, el sistema educativo debe instaurar una crítica en sus modelos pedagógicos, atendiendo ante todo al componente comunicativo y tecnológico, y la forma histórica habitual que le domina en su práctica, para ejercer sobre ésta un relevo conceptual, que experimente el abandono de su concepción instrumental, y adopte como desafío, su comprensión como agente constituyente de los procesos de potenciación juvenil. 
Por otra parte, al querer abordar los consumos culturales, dentro estos, los medios de comunicación fue posible penetrar en la cotidianeidad e identidad de los jóvenes. El lugar de dichos consumos en el tiempo libre es decisivo. Ver televisión, escuchar música, chatear en Internet y operar videojuegos son las prácticas de consumo cultural con mayor frecuencia en los usos del tiempo libre de la juventud.

Finalmente, el proyecto ha permitido evidenciar una insuficiencia de información y estadísticas sobre las y los jóvenes con que cuentan las entidades oficiales del municipio. Igualmente, el acceso a los servicios disponibles para este sector social es limitado por la falta de canales de información que intermedien entre la oferta y la demanda juvenil de educación, trabajo, cultura, recreación, entre otros.

\section{CONCLUSIONES}

Es recurrente la determinación juvenil por lograr el acceso y manejo de medios de comunicación como espacios efectivos para el acceso a la información y la visibilización de la juventud. Por ello, dicha determinación se convierte en posibilidad de concretar un futuro deseado por las y los jóvenes a través de los medios de comunicación locales.

Los medios de comunicación son un instrumento fundamental en el empoderamiento, la facilidad y disponibilidad en el acceso de información posibilita el desarrollo de un sentido crítico y participativo que la juventud reclama.

El papel que cumplen los medios de comunicación en la formulación de una Política Pública Municipal de Juventud debe superar la mera divulgación, reconociendo el papel activo que las y los jóvenes podrían ejercer a través de la opinión.

\section{REFERENCIAS BIBLIOGRÁFICAS}

BARBERO, J. (1996), Heredando el futuro. Pensar la educación desde la comunicación, Nómadas Núm. 5, Bogotá.

BARBERO, J. Retos culturales de la comunicación a la educación. Elementos para una reflexión que está por comenzar. En Comunicación, medios y educación. Un debate para la educación en democracia. Roxana Morduchowicz (Coord). Ed, Octaedro.

LÓPEZ, H (2001), Un Enfoque Histórico-Hermenéutico y Crítico-Social en Psicología y Educación Ambiental. Medellín, Colombia. Universidad Pontificia Bolivariana.

MUSITU, G. (2004), Introducción a la psicología comunitaria. Publicado por Editorial UOC. 
POLÍTICA PÚBLICA DE JUVENTUD DE CUNDINAMARCA. Derechos, Desarrollo y Política Pública: Garantías y oportunidades para los proyectos de vida individuales y colectivos de las y los jóvenes en Cundinamarca (Documento preliminar para discusión interna), Gobernación de Cundinamarca, 6 de Noviembre de 2007.

SILVA, C. Y LORETO, M. (2004), Empoderamiento: Proceso, Nivel y Contexto. PSYKHE. Vol. 13, n. ${ }^{\circ}$ 1, 29-39, Universidad Católica de Chile. 